\title{
Indicadores de calidad en el cuidado humanizado de personas con sufrimiento psíquico en etapa aguda
}

Quality Indicators in the Humanized Care of Users with Psychic Suffering in the Acute Stage

Indicadores de qualidade no atendimento humanizado a usuários com sofrimento psíquico na fase aguda

María Gabriela Dotti Ríos ${ }^{1}$, María Araceli Otárola Acosta²

\section{Resumen:}

La práctica de enfermería, incorpora el cuidado humanizado como una vía para valorar además de aspectos físicos en las personas, los elementos mentales y emocionales implicados en el contexto de la enfermedad. Los indicadores de calidad han funcionado como aspectos que son objeto de evaluación sanitaria hacia el restablecimiento de la salud afectada. Estos indicadores suelen proveer información fundamental para el desarrollo de una práctica asistencial de calidad y al mismo tiempo refieren procesos para alcanzar los objetivos trazados en la atención de los centros sanitarios. La práctica de enfermería dirigida a superar el sufrimiento psíquico en etapa aguda, requiere la valoración holística, con lo cual alcanzar un mayor impacto en la salud de la persona. El cuidado humanizado de éstas personas que sufren de forma aguda, influye sobre el afrontamiento de esta realidad compleja, el sentirse escuchados, atendidos, e impulsando su participación y autonomía en el tratamiento, propicia un mejor afrontamiento a la realidad particular de cada persona. Aunque muchos profesionales de enfermería aplican un cuidado humanizado enmarcado en la religión o política, es fundamental redireccionar acciones para empoderar a la persona. El objetivo central del artículo está dirigido a efectuar un análisis reflexivo sobre los indicadores de calidad en el cuidado humanizado de las personas con sufrimiento psíquico en etapa aguda, para valorar el trabajo desarrollado de los profesionales de enfermería y su impacto en la salud de los mismos.

\footnotetext{
${ }^{1}$ Licenciada en Enfermería. Magister. Prof. Adj. Departamento de Enfermería en Salud Mental. Facultad de Enfermería. UdelaR. ORCID: 0000-0001-7817-2054. E-mail: gabydotti@gmail.com ${ }^{2}$ Licenciada en Enfermería. Magister. Prof. Agda. Departamento de Enfermería en Salud Mental. Facultad de Enfermería. UdelaR. ORCID: 0000-0002-1006-3350. E-mail: otarolamariaraceli@gmail.com
} 
Palabras clave:

Indicadores de Calidad de la Atención de Salud, Atención de Enfermería, Estres Psicológico.

\section{Abstract:}

Nursing practice incorporates humanized care as a way to assess, in addition to physical aspects in people, the mental and emotional elements involved in the context of the disease. The quality indicators have functioned as aspects that are object of sanitary evaluation towards the reestablishment of the affected health. These indicators usually provide fundamental information for the development of a quality care practice and at the same time refer to processes to achieve the objectives set in the care of health centers. Nursing practice aimed at overcoming mental suffering in the acute stage requires holistic assessment, thereby achieving a greater impact on the health of the person. The humanized care of these people who suffer acutely, influences the coping with this complex reality, feeling heard, cared for, and promoting their participation and autonomy in the treatment, fosters a better coping with the particular reality of each person. Although many nursing professionals apply humanized care framed in religion or politics, it is essential to redirect actions to empower the person. The main objective of the article is aimed at carrying out a reflective analysis on the quality indicators in the humanized care of people with mental suffering in the acute stage, to assess the work carried out by nursing professionals and its impact on their health.

\section{Keywords:}

\section{Quality Indicators of Health Care, Nursing Care, Psychological Stress.}

\section{Resumo:}

A prática de enfermagem incorpora o cuidado humanizado como forma de avaliar, além dos aspectos físicos nas pessoas, os elementos mentais e emocionais envolvidos no contexto da doença. Os indicadores de qualidade têm funcionado como aspectos que são objeto de avaliação sanitária para o restabelecimento da saúde afetada. Esses indicadores costumam fornecer informações fundamentais para o desenvolvimento de uma prática assistencial de qualidade e, ao mesmo tempo, referem-se a processos para atingir os objetivos traçados na assistência aos centros de saúde. A prática de enfermagem voltada para a superação do sofrimento mental na fase aguda requer avaliação holística, alcançando assim um maior impacto na saúde da pessoa. O cuidado humanizado a essas pessoas que sofrem agudamente, influencia no enfrentamento dessa realidade complexa, sentindo-se ouvido, cuidado e promovendo sua participação e autonomia no tratamento, favorece um melhor enfrentamento da realidade particular de cada pessoa. Embora muitos profissionais de enfermagem apliquem o cuidado humanizado enquadrado pela religião ou pela política, é fun- 
damental redirecionar as ações para empoderar a pessoa. O objetivo principal do artigo é realizar uma análise reflexiva sobre os indicadores de qualidade no cuidado humanizado à pessoa com sofrimento mental na fase aguda, para avaliar o trabalho desenvolvido pelos profissionais de enfermagem e seu impacto na saúde.

\section{Palavras-chave:}

Indicadores de Qualidade em Assistência à Saúde, Cuidados de Enfermagem, Estresse Psicológico.

\section{Introducción}

La calidad de la asistencia de acuerdo a Organización Panamericana de la Salud (OPS), es uno de los aspectos en torno a los cuales se centran los objetivos de la mayoría de las organizaciones sanitarias, especialmente por el hecho de que gracias a ello puede contarse con la posibilidad de que las personas reciban el diagnóstico correcto y tratamiento con los cuales recuperar la salud de forma óptima ${ }^{(1)}$. Es importante valorar cada uno de los aspectos implicados en el contexto de su enfermedad, como los antecedentes familiares, diagnósticos secundarios, edad, sexo, entre otros ${ }^{(2)}$. De allí, la importancia de poner en práctica elementos de valoración con los cuales optimizar el trabajo sanitario, minimizando a su vez la posibilidad de riesgo para las personas y los profesionales de la salud.

Los aportes de Avedis Donabedian ${ }^{(3)}$ han sido reconocidos de forma significativa por su visión hacia la implementación de estrategias de evaluación de la calidad asistencial, que toma en cuenta procesos, estructura y resultados obtenidos. Gracias a ello, fue posible organizar la puesta en acto de los hospitales o centros de salud para alcanzar las metas organizacionales planteadas y ofrecer calidad de vida a las personas.
En los años 90', se implementó el Indicator Measurement System (IM System) por la Joint Commission $^{(4)}$ con la idea de evaluar indicadores relacionados con procesos y resultados derivados de ellos. Entre los aspectos interesantes que la Joint Commission tomó en cuenta fue la implementación de una agenda de cambio para valorar resultados de la atención sanitaria a punto de partida de los profesionales sanitarios que formaban parte de la atención a la salud en los centros asistenciales ${ }^{(5)}$.

Todos estos avances a nivel de la valoración de calidad asistencial fueron posibles considerando que los indicadores se estructuran para identificar aspectos que requieren ser mejorados y que en su aparición refieren situaciones no deseables para las organizaciones sanitarias, impactando sobre la calidad del trabajo desarrollado en sus espacios de atención en salud ${ }^{(3)}$. Correa explica que los indicadores de calidad tienen un propósito doble:

Primero, proveer información que sea trazadora de la calidad de la atención en el sistema y permita la referenciación a nivel nacional y/o internacional y segundo: promover procesos de mejoramiento en las instituciones para ayudar a que la 
calidad de atención en el sistema de salud alcance eficiencia y efectividad, convirtiéndose en referencia a nivel nacional e internacional ${ }^{(6)}$.

Los indicadores de calidad, hacen posible la monitorización de comportamientos del recurso humano los cuales deben estar dirigidos a mantener la seguridad en el paciente, la buena atención y acompañamiento oportuno a lo largo de los tratamientos. De esta manera, los indicadores contribuyen a la gestión de procedimientos y actividades propias de la práctica sanitaria. Es fundamental diseñar correctamente los indicadores de calidad, con los cuales trazar la adecuada ruta hacia el restablecimiento de la salud afectada ${ }^{(6)}$.

Entre las características de los indicadores de calidad, se destaca la importancia de su efectividad para la recolección de información. La aplicación de un indicador correctamente, propicia la validez y confiabilidad de sus resultados, por lo cual deben ser concretos, claros, precisos y con el menor error posible en su estructuración (7). Un indicador de calidad refiere eficiencia, continuidad, satisfacción, adecuación y otros elementos fundamentales para alcanzar calidad en la práctica asistencial desarrollada. El deseo de apoyar la salud del ser humano con una buena asistencia sanitaria se evidenció en la Teoría de Jean Watson, quien explica la necesidad de valorar al usuario como un ser integral, con necesidades, pero con capacidades para colaborar en la planificación de su cuidado asistencial (8). Al mismo tiempo, Watson ${ }^{(9)}$ explica que el cuidado precisa de un compromiso personal, moral, social e incluso espiritual por parte del profesional de enfermería, como de las personas cercanas al usuario, de allí surgió el cuidado humanizado como sinónimo de buen trato, o satisfacción por parte de la persona, comprendiendo que existe una real necesidad de cuidado a la salud por parte del individuo cuidado.

Algunos indicadores de calidad referidos al cuidado humanizado tienen que ver con la disminución del sufrimiento y son mencionados por Ariza quien ubica la categoría para valorar los sentimientos de las personas, conformación de relaciones interpersonales, intercambio comunicativo, interacción enfermera/o-paciente, información oportuna, recibir calor humano, entre otros., para impulsar sentimientos que ayuden a la sanación o restablecimiento de quien se cuida y atiende, así como del profesional de la salud ${ }^{(10)}$. Los indicadores de calidad en el cuidado humanizado, incorporan además desde la perspectiva de Ariza la categoría apoyo emocional, con el cual el profesional de enfermería identifica si la persona asume una posición activa a su tratamiento, reconoce su estado actual de la enfermedad, se le fortalece internamente, impulso a la recuperación de la vida cotidiana, entre otros elementos que permiten reconocer si se es tratado de forma humanitaria dentro del contexto sanitario ${ }^{(10)}$. En cuanto a la categoría apoyo físico, refiere indicadores para percibir si el profesional de enfermería entra en contacto con ellos de forma significativa, por tanto, se valora el cubrir las necesidades físicas, mirar a los ojos cuando se realiza el contacto, percepción de respeto por parte del profesional de enfermería, comodidad física o mental, entre otros indicadores ${ }^{(10)}$.

Otra categoría que existe es la que evalúa la proactividad de la enfermera, caracterizada por ir más allá de los requerimientos de las personas. En ella, se considera la participación o incorporación del profesional de enfermería al proceso de recuperación de las personas contemplando sus necesidades o requerimientos, especialmen- 
te ante el dolor. El desarrollo de empatía y escucha activa es parte del cuidado humanizado que los mismos necesitan ${ }^{(11)}$. En la categoría priorizar al ser cuidado, se identifica a las personas como seres únicos con pensamientos propios, sentimientos, actitudes y requerimientos sanitarios particulares; en tal sentido, el profesional de enfermería precisa visualizar como una prioridad la atención al llamado las veces que se precisen. El apoyo brindado, les permite entender que tiene un valor incalculable, por lo cual puede sentirse reconfortado, seguro y atendido como lo requiere ${ }^{(12)}$.

Tal como se evidencia, las categorías de indicadores de calidad mencionadas refieren a la atención enfermera con un componente de humanidad. El propósito es apoyar a la persona en el manejo del dolor, el tratamiento adecuado y encontrar las mejores alternativas para lograr la calidad de vida que todo ser humano desea ${ }^{(13)}$. En algunas oportunidades la persona llega a tener un malestar tan profundo que llega a entrar en sufrimiento, sin embargo, es algo que no se debe confundir con el dolor. Desde la perspectiva de Cassell ${ }^{(14)}$ el sufrimiento supone el sentirse acorralado, con un malestar que amenaza la integridad del individuo e incluso le hace sentir que puede desintegrarse emocionalmente, por ello, el sufrimiento suele ser una experiencia al límite que impacta lo existencial, moral, social, religioso e incluso lo político, llevando al individuo a reflexionar sobre su vulnerabilidad.

En algunos ámbitos sanitarios el sufrimiento se asocia con los cuidados intensivos, oncológicos o paliativos; sin embargo, esto no evita la realidad de encontrar personas que sufren y tienen dificultades para ser atendidos respecto a esta situación. Muchos de los profesionales de la salud suelen descuidarse de su rol sanitario buscando intervenir solo biológicamente hacia la curación, dejando de lado el estado psico-social, e incluso espiritual de las mismas ${ }^{(15)}$. Esta situación deshumaniza la práctica sanitaria y despersonaliza a las personas. En el contexto de la práctica en enfermería con enfoque en salud mental y psiquiatría, es fundamental estar atentos a valorar la espiritualidad del individuo, con el objetivo de incorporar elementos holísticos que amplíen el rango de acción de la atención sanitaria. En el cuidado humanizado el individuo es algo más que la suma de sus partes, aspecto que genera la reflexión de influir positivamente en la salud de las personas, impulsando resiliencia, afrontamiento y motivación a lograr calidad de vida ${ }^{(16,17)}$.

En la práctica de enfermería humanizada, especialmente hacia el cuidado del sufrimiento psíquico, se desarrolla una postura muy unida a la compasión pues refiere el apoyo sanitario de una persona a otro ser humano, que ha sido denominado relación de ayuda. El acompañamiento desarrollado se fundamenta en la escucha activa, y ante el sufrimiento del otro, el diálogo puede ser una vía para la posibilidad de cambiar la realidad del enfermo psiquiátrico; con la esperanza se encuentra sentido al trabajo desarrollado y se abre una ventana para que la persona pueda expresar en el diálogo sus temores, alegrías, u otros elementos emocionales ${ }^{(16,}$ 17). En la práctica enfermera, surge la taxonomía NANDA-NIC-NOC (NNN) como una herramienta para los planes de cuidado a los pacientes, de los cuales se destaca el NOC por cuanto tiene un carácter espiritual necesario para ofrecer calidad en el cuidado humanizado a las personas con sufrimiento psíquico ${ }^{(18,19)}$. Los indicadores de calidad NOC tienen que ver con la valoración del apoyo espiritual y el crecimiento espiritual, dentro de los cuales puedan conseguir un equilibrio a través de las creencias, po- 
der comunicarse, evocar significados, encontrar consuelo, fuerza e incluso esperanza de vida.

\section{Objetivo}

Efectuar un análisis reflexivo sobre los indicadores de calidad en el cuidado humanizado de usuarios con sufrimiento psíquico en etapa aguda, como vía para valorar el trabajo desarrollado de los profesionales de enfermería y su impacto en la salud de las personas.

\section{Reflexión}

Al profundizar en el contexto de los indicadores de calidad enmarcados en el cuidado humanizado es posible reflexionar sobre su importancia en la valoración de los sistemas sanitarios, bien sea para la toma de decisiones o para redireccionar las acciones emprendidas que no han alcanzado los niveles de calidad deseados. En efecto, los indicadores además de ofrecer información, refieren una mayor eficiencia, así como efectividad en la práctica sanitaria implementada al abrir posibilidades para ajustar la atención a la salud de las personas.

Los profesionales de la salud que aplican un cuidado humanizado a las personas con sufrimiento psíquico, ponen en práctica acciones para valorar a las mismas en sus necesidades, y limitaciones; dejan de lado la indiferencia, para poner en práctica comportamientos, actitudes y acciones hacia el restablecimiento de la salud afectada. Aunque la enfermedad produzca sufrimiento psíquico, el aplicar los indicadores de calidad hace posible estar al tanto de la situación sobre la manera como la persona valora la atención recibida y percibe el esfuerzo por apoyar su salud deteriorada. Es significativo analizar la existencia de un proceso orgánico gene- rador en el organismo de la persona y el cuadro clínico que éste genera, especialmente por el hecho de que afecta no solo en la aparición de la enfermedad, sino que también impacta en las emociones de las mismas. Los indicadores de calidad mencionados por Ariza de tipo emocional, físico, aquellos que evalúan la proactividad, el priorizar a la persona, entre otros elementos son fundamentales para apoyar el cuidado humanizado en aquellos con sufrimiento psíquico agudo, por cuanto ubica el contexto de la persona e impulsa una práctica sanitaria ajustada a la realidad del individuo, con sus necesidades particulares ${ }^{(\mathbf{1 0})}$.

En el desarrollo de enfermedades crónicas capaces de producir sufrimiento psíquico agudo, es fundamental aplicar indicadores para medir la puesta en acto de una práctica de enfermería humanizada que valore no solo la situación del organismo en su estado físico, sino aquellos aspectos emocionales que impacten negativamente sobre su equilibrio. En este contexto, el profesional de enfermería requiere estar atento al esquema personalidad y conciencia, cuya alteración propicia una desestructuración de conciencia, tipo episodios agudos que repercuten en la salud mental y emocional de las personas (19).

Es allí, donde los indicadores de salud se convierten en una guía para conocer los alcances del trabajo desarrollado y los aportes alcanzados a la salud de las personas. En tal sentido, al considerar los aspectos analizados, es posible comprender que el cuidado humanizado a la persona con sufrimiento psíquico en etapa aguda pasa por la puesta en acto de un trabajo de atención a la salud sin barreras o estigmas, promoviendo el ser respetuosos, justos y benévolos con estos seres humanos que pasan por una situación difícil en sus vidas. El desarrollo 
de un compromiso basado en el apego a la moral y respeto por los demás, capaz de acompañar la evolución o deterioro de la salud de los individuos, los gestos, comunicación, postura, miradas y todos aquellos elementos que forman parte del cuidado humanizado tan necesario en los espacios sanitarios.

\section{Conclusiones}

- Los distintos elementos analizados sobre los indicadores de calidad en el cuidado humanizado de las personas con sufrimiento psíquico en etapa aguda, como vía para valorar el trabajo desarrollado de los profesionales de enfermería y su impacto en la salud de los pacientes, mostraron el cumplimiento del propósito planteado dentro del análisis reflexivo desarrollado.

- Los indicadores de calidad contribuyen a monitorear comportamientos del recurso humano sanitario que tiene como labor efectuar una atención integral, brindar seguridad a las personas, acompañarlas a lo largo de sus tratamientos. Los indicadores de calidad refieren excelentes oportunidades para trazar una ruta adecuada con la cual apoyar al establecimiento de la salud de quien así lo requiere ${ }^{(6)}$.

- El cuidado a la salud por parte de los trabajadores sanitarios, requiere compromiso moral, aspectos sociales, espirituales y personales, a partir de lo cual se estructura un cuidado humanizado en el que prevalece en el usuario la sensación de ser bien atendidos, escuchados y tratados. El cuidado humanizado es una forma de impulsar un clima organizacional idóneo dentro de los centros de salud, bien sea porque las personas encuentran satisfacción por el trato recibido o porque el personal de salud puede observar directamente la real necesidad de una atención integral a las mismas.

- En el cuidado humanizado, los indicadores de calidad apoyan la valoración del intercambio comunicativo, la información oportuna, el reconocimiento y respeto por los sentimientos de las personas, el desarrollo de interacciones interpersonales en sus distintas vertientes, entre otros aspectos ${ }^{(10)}$. A partir de ello, es posible apoyar a que los usuarios sientan desde una perspectiva humanista, su valor como individuo, sensibilidad por su dolor, etc.

- Los indicadores de calidad, toman en cuenta la categoría apoyo emocional, la cual trae consigo la oportunidad de que la persona participe activamente en su tratamiento, fortaleciéndolo internamente e impulsando la valoración de sus temores, sentimientos e incluso frustraciones. Con estos indicadores es posible identificar si el trato brindado en la atención en salud es humanitario o no. ${ }^{(10)}$.

- Con los indicadores de calidad, el cuidado humanizado es visualizado desde una postura sistémica, en la que el objetivo central de los procesos e interacciones es la salud de las personas. Con resiliencia, motivación e impulso al afrontamiento desde una perspectiva positiva, es posible apoyar al restablecimiento efectivo de la salud de los mismos ${ }^{(17)}$. Además, existe un elemento de espiritualidad implicado en el cuidado humanizado, que va más allá del solo hecho de aplicar un tratamiento médico; el propósito es conformar un entramado de interacciones humanizadas con el cual impulsar al recurso humano sanitario como un personal motivado, con sentido de pertenencia y cariño por la labor ejercida ${ }^{(17)}$. 
- La NANDA-NIC-NOC (NNN) es una herramienta que apoya la planificación para el cuidado de las personas. Sugiere un enfoque humanista en el cuidado al otro, se basa en acciones de calidad que impacten positivamente en el sufrimiento psíquico. Es decir, refiere espacios para la valoración de un cuidado donde el apoyo espiritual esté presente, especialmente en cuanto al lograr un balance entre la comunicación, la esperanza de vida, la fuerza y la evocación de significados en las personas ${ }^{(18)}$.

- El cuidado humanizado muestra una tendencia hacia la disminución del sufrimiento psíquico no solo físico, incorporando estrategias con las cuales apoyar en las necesidades, así como limitaciones de las personas. Entre los elementos que precisan ser eliminados para ofrecer una atención de calidad están el distanciamiento con las personas, indiferencia por su estado de salud, desapego por la labor ejercida, y transformarla por prácticas comprensivas, atentas a los procedimientos y tratamientos sanitarios, escucha activa, comprensión de que cada persona tiene sus propias necesidades, así como situaciones particulares, entre otros (19).

- En el caso de personas con sufrimiento psíquico en etapa aguda, el cuidado humanizado elimina barreras o estigmas hacia una atención de calidad. Para ello, el profesional de la salud precisa ser benévolo, atento y comunicativo con el quien cuida, comprendiendo la difícil situación por la que está pasando ese ser humano. En el momento en que el desenvolvimiento del profesional de enfermería indica atención a la evo- lución o deterioro de la persona, se inicia una cadena en la que se fortalece la atención a la salud, especialmente si pone en práctica un cuidado humanizado. Ante ello, los indicadores de calidad, pueden proveer la valoración objetiva y en el momento preciso para conocer los alcances obtenidos, o redireccionar la atención brindada, especialmente en el caso de que se haya distanciado de un cuidado humanizado. El propósito es trabajar de forma equilibrada, desde una postura humana y comprensiva, sobre las bases de una práctica enfermera actualizada y preparada profesionalmente para apoyar los tratamientos sanitarios indicados.

- En efecto, la ocurrencia de enfermedades crónicas en su mayoría produce sufrimiento agudo, por ello, la práctica humanizada es una opción para minimizar el impacto de la enfermedad; sin embargo, es muy común evidenciar que no siempre se pone en práctica, por ello, los indicadores de calidad refieren una excelente oportunidad para evaluar la puesta en acto del cuidado humanizado.

- La práctica de enfermería humanizada, muestra buenas posibilidades para comprender la situación del individuo en su estado físico y apoyarle a nivel psicosocial, pues es de esta manera en que se balanceará la situación hacia el equilibrio necesario para disminuir el dolor, la angustia e incluso la incomprensión de lo que ocurre con el organismo. 
1. Organización Panamericana de la Salud. Clasificación estadística internacional de enfermedades y problemas relacionados con la salud. 10 . ed. Washington: OPS; 2016. v.2.

2. Alcázar Casanova F, Iglesias Alonso F. Historia y definición de la calidad de los servicios sanitarios. En: Servicio de Salud de Castilla-La Mancha. Manual de calidad asistencial. [Internet]. 2009. Disponible en: https://sanidad.castillalamancha. es/sites/sescam.castillalamancha.es/files/ documentos/pdf/20131017/manual_calidad_asistencial_de_la_seca.pdf [consulta: 8 ene. 2021].

3. Rodríguez Pérez MP. Grande Armesto M. Calidad asistencial: concepto, dimensiones y desarrollo operativo. [Internet]. 2014. Disponible en: http://e-spacio.uned.es/fez/ eserv/bibliuned:500957/n14-1_Calidad_ asistencial.pdf [consulta: 8 ene. 2021].

4. Joint Commission on Accreditation of Healthcare Organizations. Primer on Indicator Development and Application: measuring quality in health care. Chicago: JCAHO; 1990.

5. Zamora-Soler JA, Maturana-Ibáñez V. El análisis de la calidad asistencial desde la perspectiva de la satisfacción y la experiencia del paciente: una mirada enfermera. Rev. Colomb. Enferm. [Internet] 2019; 18(2): e010. Disponible en: https://doi. org/10.18270/rce.v18i2.2513 [consulta: 8 ene. 2020].

6. Colombia. Ministerio de la Protección Social. Observatorio de Calidad de la Atención en Salud. Biblioteca nacional de indicadores de calidad de atención en salud.
[Internet]. 2011. Disponible en: https:// es.calameo.com $/ \mathrm{read} / 000147616146 \mathrm{fbc}$ f6bc15 [consulta: 10 ene. 2020].

7. Davins Miralles J. Metodología de evaluación y mejora continuada: la medida de la calidad, monitorización, indicadores, calidad y estadística. [Internet]. 2011. Disponible en: http://projectes.camfic.cat/CAMFiC/Seccions/GrupsTreball/Docs/Qualitat/TEMA1.pdf [consulta: 10 ene. 2020].

8. Ugarte Chang JA. Características del cuidado humanizado que brinda la enfermera según las personas viviendo con VIH en estadio SIDA. [Tesis de maestría]. [Internet]. 2015. Disponible en: https:// repositorio.upch.edu.pe/bitstream/handle/20.500.12866/1038/Caracteristicas_ UgarteChang_Jenny.pdf?sequence $=1 \&$ is Allowed $=y$ [consulta: 15 mar. 2020].

9. Urra ME, Jana AA, García VM. Algunos aspectos esenciales del pensamiento de Jean Watson y su teoría de cuidados transpersonales. Ciencia y Enfermería [Internet] 2011; 17(3):11-22. Disponible en: https://www.scielo.cl/pdf/cienf/v17n3/art02. pdf [consulta: 11 mar. 2020].

10. Ariza Olarte C. Soluciones de humanización en salud en la práctica diaria. Enfermería Universitaria ENEO-UNAM [Internet] 2012; 9(1): 45-51. Disponible en: http://www.scielo.org.mx/pdf/eu/v9n1/ v9n1a6.pdf [consulta: 4 mar. 2020].

11. Marriner-Tomey A, Alligood RM. Modelos y teorías en enfermería. $5^{\mathrm{a}}$. ed. Madrid: Mosby; 2003.

12. Guzmán TS. Cuidado humano en la formación del Estudiante de Enfermería según 
la Teoría de Jean Watson, Chiclayo, Perú 2011. [Tesis de maestría]. [Internet]. 2013. Disponible en: https://tesis.usat.edu.pe/ bitstream/20.500.12423/571/1/TM_Guzman_Tello_SocorroMartina.pdf [consulta: 4 mar. 2020].

13. Muñoz Hernández Y, Coral Ibarra R, Moreno Prieto D, Pinilla Pinto D, Suárez Rodríguez Y. Significado del cuidado humanizado en egresadas de la facultad de enfermería. Repert Med Cir [Internet] 2009; 18(4): 246-50. Disponible en: https://revistas.fucsalud.edu.co/index.php/repertorio/article/view/561/601 [consulta: 4 mar. 2020].

14. Cassell EJ. The nature of suffering. $2^{\circ} \mathrm{ed}$. Oxford: Oxford University Press; 2003.

15. Caldeira S, Castelo Branco Z, Vieira M. A espiritualidade nos cuidados de enfermagem: revisão da divulgação científica em Portugal. Rev Enf Ref [Internet] 2011; 3(5): 145-52. Disponible en: http://www.index-f. com/referencia/2011pdf/35-145.pdf [consulta: 07 ene. 2020].
16. Lander R. Un estudio sobre el sufrimiento humano. Psicoanálisis [Internet] 2012; 24(1): 111-16. Disponible en: https:// dialnet.unirioja.es/servlet/articulo?codigo $=4243778$ [consulta: 7 ene. 2020].

17. Guirao-Goris JA. La espiritualidad como dimensión de la concepción holística de salud. ENE. Revista de Enfermería [Internet] 2014; 7(1). Disponible en: http:// ene-enfermeria.org/ojs/index.php/ENE / article/view/252 [consulta: 07 ene. 2020].

18. Moorhead S, Jonhson M, Maas ML, Swanson E. Clasificación de resultados de enfermería (NOC). 4ª ed. Barcelona: Elsevier; 2009.

19. Martins JJ, Backes DS, Cardoso RS, Erdmann AL, Albuquerque GL. Resignificando la humanización desde el cuidado en el curso de vivir humano. Rev enferm UERJ [Internet] 2008; 16(2): 276-81. Disponible en: https://docplayer.es/80877618-Resignificando-la-humanizacion-desde-el-cuidado.html [consulta: 6 feb. 2020].

Recibido: 12.04.21

Aceptado: 07.07.21 